\title{
Consistent incremental approximation of dissipation pseudo-potentials in the variational formulation of thermo-mechanical constitutive updates
}

\author{
L. Stainier \\ Institut de recherche en Génie Civil et Mécanique (GeM - UMR 6183 CNRS) Ecole Centrale de Nantes, 1 rue de la Noë, BP 92101 , F-44321 Nantes, France
}

In this paper, we detail a consistent approximate expression for incremental dissipation pseudopotentials which appear in the variational formulation of coupled thermo-mechanical boundary-value problems. We explain why the most intuitive expression does not work in the case of an explicit temperature dependence in the dissipation, and propose an alternative expression ensuring consistent results when reducing the time increment towards zero.

\section{Keywords:}

Variational constitutive updates Finite thermo-visco-plasticity

Thermomechanics

\section{Introduction}

Thermo-mechanical coupling effects are of importance in a number of engineering problems: e.g. manufacturing processes such as forging or welding, or when considering the dynamic behavior of materials such as metals or polymers. Coupling between mechanical and thermal responses can occur through different mechanisms: temperature dependence of elastic and visco-plastic properties, heating from dissipative micromechanisms, phase change,... These effects can be introduced in constitutive models and balance equations (momentum, energy), but this breaks the mathematical symmetry of the problem. As a result, numerical models derived by standard approaches such as finite elements (Oden, 1972; Argyris and Doltsinis, 1981) are thus non-symmetric as well, which incurs higher computational cost and possibly some loss in robustness of solvers. In order to avoid these difficulties, partitioned or staggered approaches have been proposed (Farhat et al., 1991; Armero and Simo, 1992,1993; Simo and Miehe, 1992), which retain the symmetry properties of isolated mechanical and thermal problems.

An energy-based variational formulation of general coupled thermo-mechanical boundary-value problems, including nonlinear and dissipative behaviors, has recently been proposed by Yang et al. (2006). In that formulation, incremental balance equations (conservation of momentum and energy) are derived as Euler-Lagrange equations of a global optimization problem. This result in a fully coupled, yet symmetric, generic framework for coupled thermomechanical problems. This formulation has for example been successfully applied to finite thermo-visco-plasticity (Stainier and Ortiz, 2010). We refer the reader to these two papers, and references therein, for a detailed description of the variational formulation and a general discussion about solving coupled thermo-mechanical boundary value problems and integrating associated constitutive equations. We will focus here on a specific aspect of the variational formulation in its incremental form.

The variational formulation is based on a thermodynamic approach to constitutive modeling, closely following the Generalized Standard Materials approach of Halphen and Nguyen (1975). In this thermodynamic approach, the constitutive behavior is described through potentials (state functions) and dissipation pseudo-potentials. These pseudo-potentials are not state functions, but are introduced in order to guarantee, through their convexity, a priori satisfaction of the second principle of thermodynamics (non-negative total dissipation). The functional which is optimized by the variational formulation is incremental by nature, and is obtained by integrating over a time step some combination of potentials (actually rate thereof) and pseudo-potentials, yielding a quantity homologous to an energy. The state functions can be integrated exactly, but not the dissipation pseudo-potentials, resulting in some level of error, which is inherent to most incremental approximations. This approximation error is acceptable as long as the formulation remains consistent with the continuous rate equations of the original constitutive model as the time step (or more generally load increment) tends to zero.

In the case of thermo-mechanical constitutive models including thermal softening effects on the yield or viscous stress, for exam- 
ple, deriving a consistent approximation to associated dissipation pseudo-potentials proves to be not as trivial as initially expected. In this paper, we illustrate this problem and then propose a consistent approximate expression for such incremental dissipation pseudo-potentials. The results obtained are illustrated on a simple example.

\section{Variational constitutive updates}

\subsection{Simplified framework}

Let us consider a simple elasto-visco-plastic model, without any hardening, and with a Perzyna-type rate-dependency. For the sake of conciseness, we will further limit ourselves to the unidimensional case, with linearized kinematics and assuming monotonous loading. We first introduce a Helmholtz free energy density

$W\left(\varepsilon, \theta, \varepsilon^{p}\right)=W^{e}\left(\varepsilon-\varepsilon^{p}, \theta\right)+W^{h}(\theta)$

where $\varepsilon$ is the total strain, $\theta$ the relative temperature (the absolute temperature is given by $T=T_{0}+\theta$, where $T_{0}$ is a reference temperature), $\varepsilon^{p}$ the plastic strain. The elastic and thermal stored energy densities $W^{e}$ and $W^{h}$ are typically taken as quadratic expressions of their (first) argument, but the actual choice has no effect on the subsequent discussion. Note that, for the sake of clarity and conciseness, we have made the simplifying assumption that there is no plastic stored energy. Accounting for such mechanisms through an additional plastic stored energy density $W^{p}\left(\varepsilon^{p}, \theta\right)$ does not modify the following discussion. Thermodynamic forces conjugate to strain and temperature are respectively the stress and the entropy:

$\sigma \equiv W_{, \varepsilon}\left(\varepsilon, \theta, \varepsilon^{p}\right)$

$\eta \equiv-W_{, \theta}\left(\varepsilon, \theta, \varepsilon^{p}\right)$

The set of constitutive equations is closed by introducing a kinetic relation for the internal variable $\varepsilon^{p}$ through the convex dissipation pseudo-potential $\psi^{*}\left(\dot{\varepsilon}^{p} ; \theta\right)$ :

$W_{,{ }_{\varepsilon}}\left(\varepsilon, \theta, \varepsilon^{p}\right)+\psi_{, \dot{\varepsilon}^{p}}^{*}\left(\dot{\varepsilon}^{p} ; \theta\right)=0$

The use of a semi-column in the expression of $\psi^{*}$ denotes a potential parametric dependence on temperature. In order to fix ideas, we will assume that the dissipation pseudo-potential consists of a rate-independent and a rate-dependent part:

$$
*\left(\dot{\varepsilon}^{p} ; \theta\right)=\sigma_{y}(\theta) \dot{\varepsilon}^{p}+\frac{m}{m+1} \sigma_{v}(\theta) \dot{\varepsilon}_{0}\left(\frac{\dot{\varepsilon}^{p}}{\dot{\varepsilon}_{0}}\right)^{(1 / m)+1}
$$

where $\sigma_{y}(\theta)$ and $\sigma_{v}(\theta)$ are respectively temperature-dependent yield and viscous stresses, while $\dot{\varepsilon}_{0}$ is a reference strain-rate and $m \in[1,+\infty$ [ a rate-dependency exponent.

\subsection{Variational update}

Consider a time increment $\left[t_{n}, t_{n+1}\right]$, for which the initial state $\left\{\varepsilon_{n}, \theta_{n}, \varepsilon_{n}^{p}\right\}$ is known as well as the final values $\varepsilon_{n+1}$ and $\theta_{n+1}$. In order to update the plastic strain $\varepsilon_{n+1}^{p}$, the following minimization problem can be solved:

$W_{n}\left(\varepsilon_{n+1}, \theta_{n+1} ; \varepsilon_{n}, \theta_{n}, \varepsilon_{n}^{p}\right) \equiv \inf _{\varepsilon_{n+1}^{p}}\left[W\left(\varepsilon_{n+1}, \theta_{n+1}, \varepsilon_{n+1}^{p}\right)\right.$
$\left.-W\left(\varepsilon_{n}, \theta_{n}, \varepsilon_{n}^{p}\right)+\eta_{n}\left(\theta_{n+1}-\theta_{n}\right)+\int_{t_{n}}^{t_{n+1}} *\left(\frac{T_{n+1}}{T_{n}} \frac{\Delta \varepsilon^{p}}{\Delta t} ; \theta(t)\right) d t\right]$

where $\eta_{n}=-W_{, \theta}\left(\varepsilon_{n}, \theta_{n}, \varepsilon_{n}^{p}\right)$, and $\Delta \varepsilon^{p}=\varepsilon_{n+1}^{p}-\varepsilon_{n}^{p}$. The stationarity (or Euler-Lagrange) equation corresponding to minimization principle (5) writes:

$W_{, \varepsilon}\left(\varepsilon_{n+1}, \theta_{n+1}, \varepsilon_{n+1}^{p}\right)+\frac{1}{\Delta t} \int_{t_{n}}^{t_{n+1}} \psi_{, \dot{\varepsilon}^{p}}^{*}\left(\frac{T_{n+1}}{T_{n}} \frac{\Delta \varepsilon^{p}}{\Delta t} ; \theta(t)\right) d t=0$

If there is no explicit dependence of dissipation pseudo-potential

* to temperature, (6) reduces to a consistent incremental approximation to (3):

$W_{, \varepsilon}\left(\varepsilon_{n+1}, \theta_{n+1}, \varepsilon_{n+1}^{p}\right)+\frac{T_{n+1}}{T_{n}} \psi_{, \dot{\varepsilon}^{p}}^{*}\left(\frac{T_{n+1}}{T_{n}} \frac{\Delta \varepsilon^{p}}{\Delta t}\right)=0$

In the general case the second term in the left-hand side is replaced by an average over the time step. Consistency with (3) is also clearly verified in that case.

\subsection{Adiabatic heat equation}

A significant advantage of the variational formulation is that the incremental energy resulting from the minimization (5) acts itself as a potential for total strain and temperature:

$$
\begin{aligned}
& \frac{d W_{n}}{d \varepsilon_{n+1}}\left(\varepsilon_{n+1}, \theta_{n+1} ; \varepsilon_{n}, \theta_{n}, \varepsilon_{n}^{p}\right)=\sigma_{n+1} \\
& \frac{d W_{n}}{d \theta_{n+1}}\left(\varepsilon_{n+1}, \theta_{n+1} ; \varepsilon_{n}, \theta_{n}, \varepsilon_{n}^{p}\right)=-\eta_{n+1}+\eta_{n} \\
& \quad+\Delta t \frac{d}{d \theta_{n+1}}\left\langle *\left(\frac{T_{n+1}}{T_{n}} \frac{\Delta \varepsilon^{p}}{\Delta t} ; \theta(t)\right)\right\rangle_{\Delta t}
\end{aligned}
$$

where $\sigma_{n+1}$ and $\eta_{n+1}$ have to be understood as evaluations of (2a) and (2b) at time $t_{n+1}$, while the \langle\rangle brackets denote an average over the time step. Using (8b), the local adiabatic heat equation can then be written, in incremental form, as

$\frac{d W_{n}}{d \theta_{n+1}}\left(\varepsilon_{n+1}, \theta_{n+1} ; \varepsilon_{n}, \theta_{n}, \varepsilon_{n}^{p}\right)=0$

In order to see this, consider first the case where there is no explicit temperature dependence of $\psi^{*}$. Noting that $\sigma=-W_{, \varepsilon}\left(\varepsilon, \theta, \varepsilon^{p}\right)$, relation (9) then indeed yields

$-\Delta \eta+\frac{1}{T_{n+1}} \sigma_{n+1} \Delta \varepsilon^{p}=0$

which is clearly consistent with the continuous adiabatic heat equation (in entropy form) $T \dot{\eta}=\sigma \dot{\varepsilon}^{p}$. In the general case, it is not always possible to obtain a closed-form expression for the average of the dissipation pseudo-potential appearing in (8b). It is then necessary to resort to some incremental approximation, which is required to lead to a consistent incremental heat equation.

\subsection{Consistent incremental approximation of dissipation}

The most intuitive approximation to the average dissipation pseudo-potential may be to take a fully implicit approach:

$\left\langle *\left(\frac{T_{n+1}}{T_{n}} \frac{\Delta \varepsilon^{p}}{\Delta t} ; \theta(t)\right)\right\rangle_{\Delta t} \approx \psi^{*}\left(\frac{T_{n+1}}{T_{n}} \frac{\Delta \varepsilon^{p}}{\Delta t} ; \theta_{n+1}\right)$

Considering the specific expression given in (4), the incremental adiabatic heat Eq. (9) yields

$$
\begin{gathered}
\Delta \eta=\frac{\Delta \varepsilon^{p}}{T_{n}}\left[\left(\sigma_{y}\left(\theta_{n+1}\right)+\sigma_{y}^{\prime}\left(\theta_{n+1}\right) T_{n+1}\right)+\sigma_{v}\left(\theta_{n+1}\right)\right. \\
\left.+\frac{m}{m+1} \sigma_{v}^{\prime}\left(\theta_{n+1}\right) T_{n+1}\left(\frac{T_{n+1}}{T_{n}} \frac{\Delta \varepsilon^{p}}{\Delta t \dot{\varepsilon}_{0}}\right)^{1 / m}\right]
\end{gathered}
$$

This incremental expression is not consistent with the continuous heat equation, because of the terms in $\sigma_{x}^{\prime}\left(\theta_{n+1}\right) T_{n+1}$. The problem 


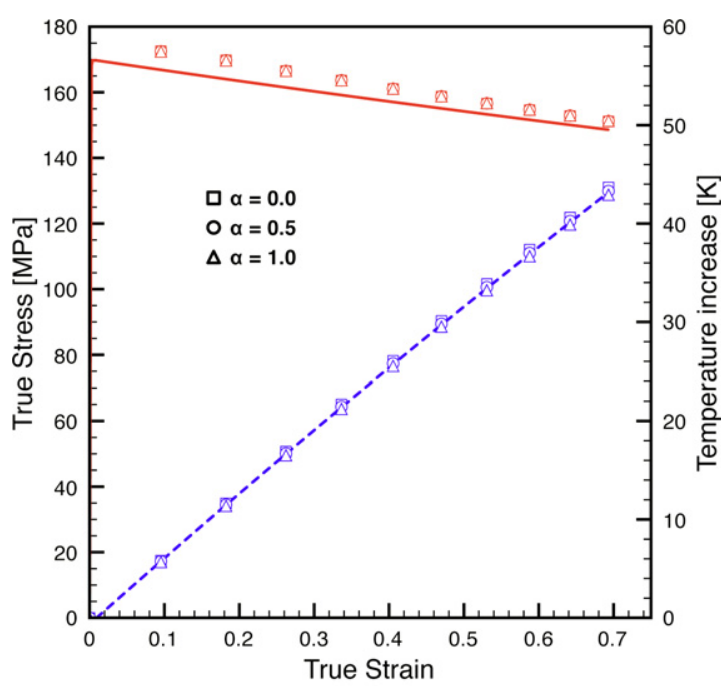

(a) slow loading rate

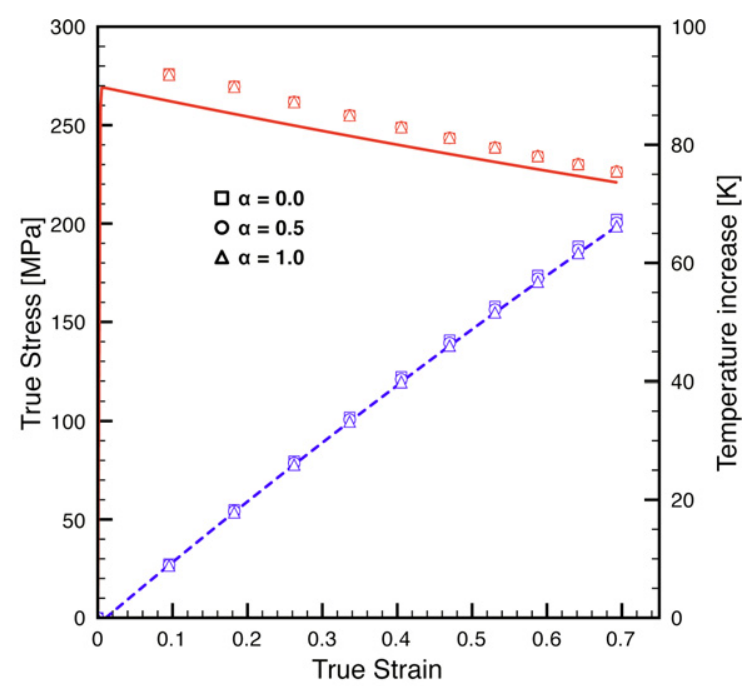

(b) fast loading rate

Fig. 1. Stress-strain (solid line) and temperature-strain (dashed line) curves under uniaxial tension (lines: 500,000 steps, symbols: 10 steps).

is not solved by taking a generalized mid-point rule (except maybe for a fully explicit approximation, which is too restrictive). An alternative expression thus has to be found.

Let us now consider the following approximation:

$$
\begin{aligned}
& \left\langle *\left(\frac{T_{n+1}}{T_{n}} \frac{\Delta \varepsilon^{p}}{\Delta t} ; \theta(t)\right)\right\rangle_{\Delta t} \approx \frac{T_{n}}{T_{n+1}} *\left(\frac{T_{n+1}}{T_{n}} \frac{\Delta \varepsilon^{p}}{\Delta t} ; \theta_{n}\right) \\
& +\frac{\Delta T}{T_{n+1}} *\left(\frac{T_{n+1}}{T_{n}} \frac{\Delta \varepsilon^{p}}{\Delta t} ; \theta_{n+\alpha}\right)
\end{aligned}
$$

where $\alpha \in[0,1]$ is an algorithmic parameter. The stationarity equation of the minimization problem then writes:

$$
\begin{gathered}
W_{, \varepsilon}\left(\varepsilon_{n+1}, \theta_{n+1}, \varepsilon_{n+1}^{p}\right)+\psi_{, \dot{\varepsilon} p}^{*}\left(\frac{T_{n+1}}{T_{n}} \frac{\Delta \varepsilon^{p}}{\Delta t} ; \theta_{n}\right) \\
+\frac{\Delta T}{T_{n}} \psi_{, \dot{\varepsilon}^{p}}^{*}\left(\frac{T_{n+1}}{T_{n}} \frac{\Delta \varepsilon^{p}}{\Delta t} ; \theta_{n+\alpha}\right)=0
\end{gathered}
$$

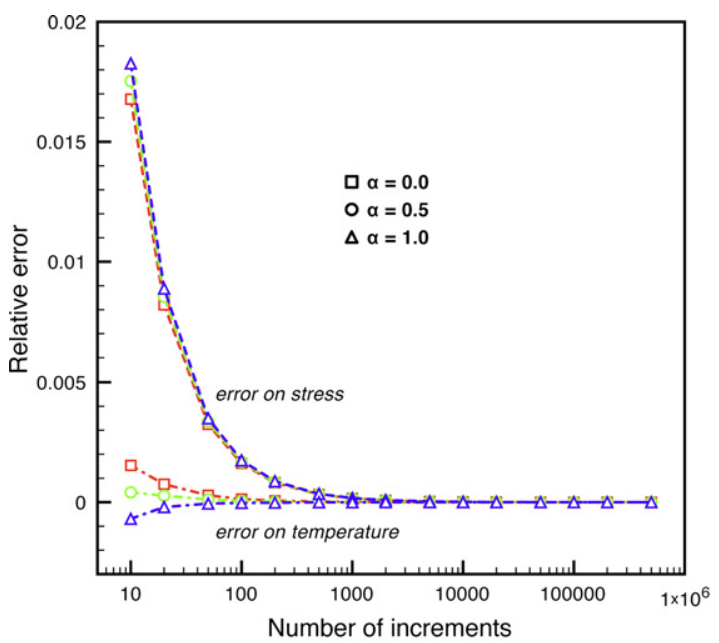

(a) slow loading rate which is consistent with (3) as $\Delta t \rightarrow 0$. The incremental adiabatic heat Eq. (9) yields

$$
\begin{aligned}
\Delta \eta= & \frac{1}{T_{n+1}}\left\{\sigma_{n+1} \Delta \varepsilon^{p}+\Delta t \frac{T_{n}}{T_{n+1}}\left(*\left(\frac{T_{n+1}}{T_{n}} \frac{\Delta \varepsilon^{p}}{\Delta t} ; \theta_{n+\alpha}\right)\right.\right. \\
& \left.\left.\left.-\psi^{*}\left(\frac{T_{n+1}}{T_{n}} \frac{\Delta \varepsilon^{p}}{\Delta t} ; \theta_{n}\right)\right)+\alpha \Delta T \quad{ }_{, \theta}^{*}\left(\frac{T_{n+1}}{T_{n}} \frac{\Delta \varepsilon^{p}}{\Delta t} ; \theta_{n+\alpha}\right)\right]\right\}
\end{aligned}
$$

where we have used (14). It is easily checked that this expression is consistent with the continuous adiabatic heat equation when $\Delta t \rightarrow 0$.

\section{Example}

In order to illustrate the above developments, we will consider an uniaxial tension test, using tridimensional elastovisco-plasticity model accounting for finite strains. This model is

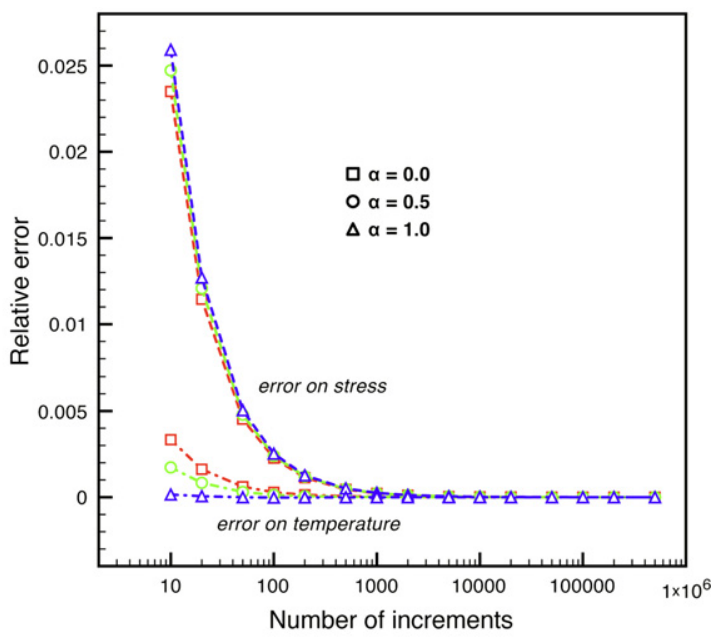

(b) fast loading rate

Fig. 2. Evolution of relative errors on final values of stress and temperature. 


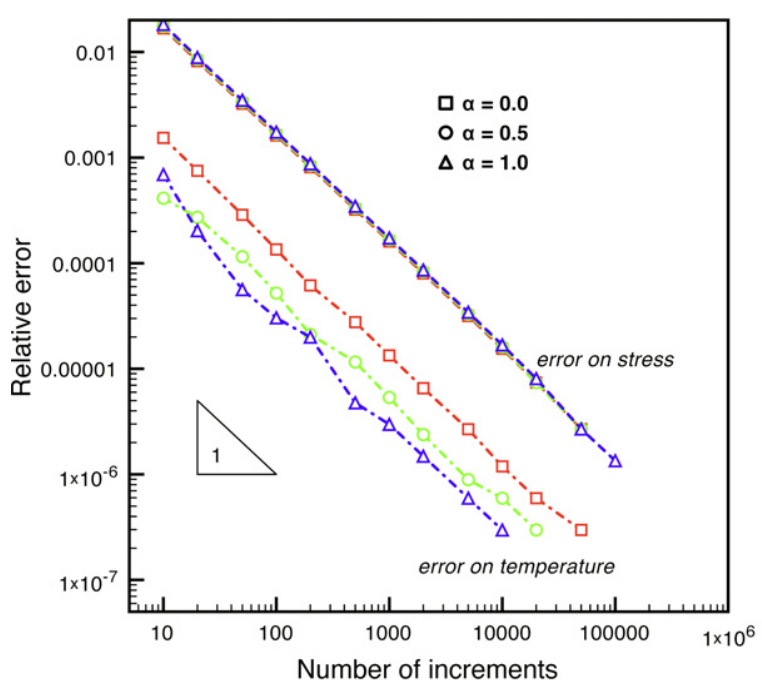

(a) slow loading rate

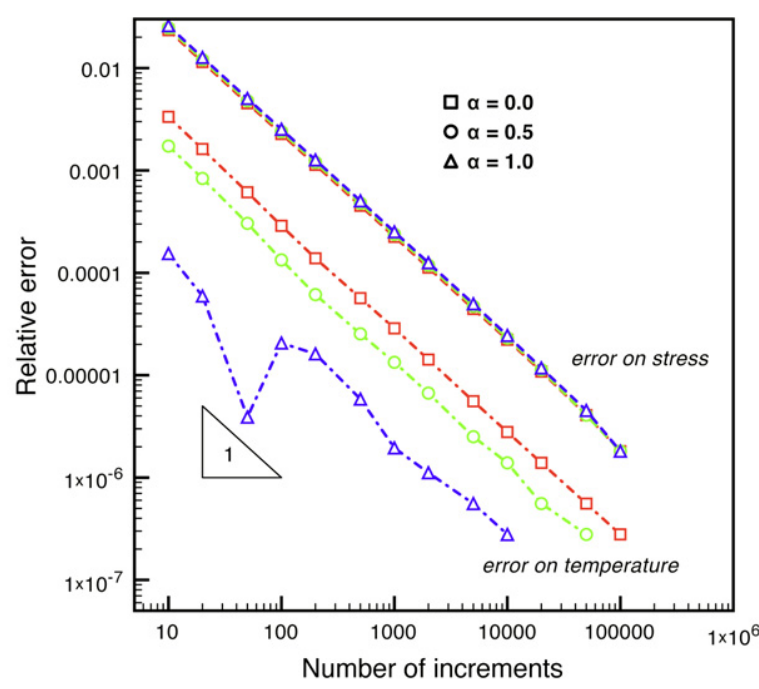

(b) fast loading rate

Fig. 3. Evolution of relative errors (in absolute value) on final values of stress and temperature.

described by a Helmholtz free energy density of the type

$W\left(\boldsymbol{F}, T, \boldsymbol{F}^{p}\right)=W^{e}\left(\boldsymbol{C}^{e}, T\right)+W^{h}(T)$

where $\boldsymbol{F}$ and $\boldsymbol{F}^{p}$ are the total and plastic gradients of deformation, $T$ the absolute temperature, and $\boldsymbol{C}^{e}=\boldsymbol{F}^{e T} \boldsymbol{F}^{e}$ the elastic right Cauchy-Green stretch tensor (with $\boldsymbol{F}^{e}=\boldsymbol{F F}^{p^{-1}}$ ). The thermo-elastic and heat capacity potentials are respectively given by

$$
\begin{aligned}
W^{e}\left(\boldsymbol{C}^{e}, T\right)= & \frac{K_{0}}{2}\left(\operatorname{tr}\left[\varepsilon^{e}\right]\right)^{2}+G_{0}\left(\operatorname{dev}\left[\varepsilon^{e}\right]\right)^{2} \\
& -\frac{3}{2} \beta\left(T-T_{0}\right) K_{0} \log \left(\operatorname{det}\left[\boldsymbol{C}^{e}\right]\right)
\end{aligned}
$$

$\left.W^{h}(T)=\rho_{0} c_{0} \quad T-T \log \left(\frac{T}{T_{0}}\right)\right]$

with the natural elastic strain defined as $\varepsilon^{e}=\frac{1}{2} \log \left(\boldsymbol{C}^{e}\right)$. Values for material parameters $K_{0}, G_{0}, \beta, \rho_{0} c_{0}$ are given in Table 1 . The dissipation pseudo-potential is given by expression (4) with $\dot{\varepsilon}^{p}=$ $\sqrt{\frac{2}{3} \boldsymbol{D}^{p} \cdot \boldsymbol{D}^{p}}$, where $\boldsymbol{D}^{p}=\operatorname{sym} \dot{\boldsymbol{F}}^{p} \boldsymbol{F}^{p^{-1}}$. The yield stress and viscous stress vary linearly with temperature:

$\sigma_{y}(\theta)=\sigma_{y 0}\left(1-\omega_{y} \theta\right)$

$\sigma_{\nu}(\theta)=\sigma_{\nu 0}\left(1-\omega_{\nu} \theta\right)$

Reference temperature is the initial temperature $T_{0}=293 \mathrm{~K}$.

A series of virtual adiabatic tensile tests were performed at strain rates of $\dot{\varepsilon}=10^{-1} \mathrm{~s}^{-1}$ (slow) and $\dot{\varepsilon}=10^{2} \mathrm{~s}^{-1}$ (fast), varying algorithmic parameters such as $\alpha$ and the time step $\Delta t$. Fig. 1 illustrates stress-strain curves obtained using very small time steps and relatively large time steps. Fig. 1 also illustrates associated temperature increase under adiabatic conditions.

Considering the solutions obtained with very small time steps (500,000 increments for a relative elongation of $100 \%)$ as the refer-

\section{Table 1}

Material parameters.

\begin{tabular}{lllll}
\hline$K_{0}[\mathrm{GPa}]$ & $G_{0}[\mathrm{GPa}]$ & $\beta\left[\mathrm{K}^{-1}\right]$ & $\sigma_{y 0}[\mathrm{MPa}]$ & $\omega_{y}\left[\mathrm{~K}^{-1}\right]$ \\
\hline 58.333 & 26.923 & $23.8 \times 10^{-6}$ & 70.0 & 0.002 \\
$\rho_{0} c_{0}\left[\mathrm{~J} \mathrm{~m}^{-3} \mathrm{~K}^{-1}\right]$ & $\dot{\varepsilon}_{0}\left[\mathrm{~s}^{-1}\right]$ & $m$ & $\sigma_{v 0}[\mathrm{MPa}]$ & $\omega_{v}\left[\mathrm{~K}^{-1}\right]$ \\
\hline $2.43 \times 10^{6}$ & 0.1 & 10.0 & 100.0 & 0.002 \\
\hline
\end{tabular}

ence (convergence to 7 significative digits was observed), we have computed errors on final values of stress and temperature for various time step sizes, in order to analyze the influence of algorithmic parameter $\alpha$ on convergence behavior. Fig. 2 shows the evolution of the relative errors on stress and temperature for slow and fast loading rates, in semi-logarithmic plots, while Fig. 3 shows the evolution of the same relative errors, but this time in absolute value, on fully logarithmic plots.

From Fig. 2, it can be seen that the relative error on temperature is typically one order of magnitude smaller than the error on stress. It also clearly appears that the error on stress monotonically converges to zero as the number of increments increases. In the case of the error on temperature, the situation is less clear: when the algorithmic parameter $\alpha=1$, a non-monotonous convergence is observed. Due to the highly non-linear nature of the thermo-mechanical coupling, no clear explanation for this behavior could be identified. Logarithmic plots in Fig. 3 show linear convergence for all quantities, independently of the choice of algorithmic parameter. The apparent super-convergence observed on temperature for a fast loading rate and $\alpha=1$ is related to the non-

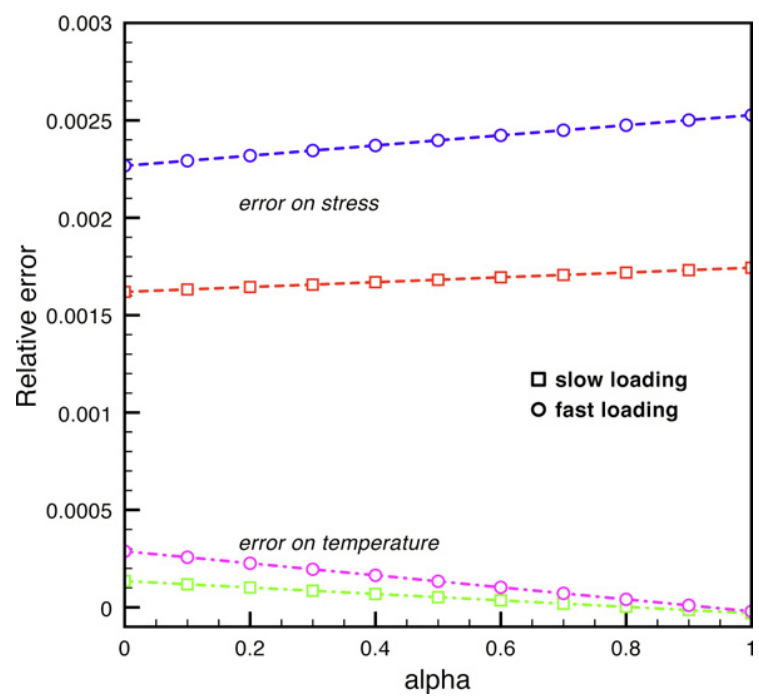

Fig. 4. Evolution of relative errors on final values of stress and temperature (obtained with 100 increments). 
monotonous convergence and the change of sign of the error. Finally, the effect of algorithmic parameter $\alpha$ on the precision can be analyzed. As can be seen from Figs. 3 and 4, this parameter shows a much less significant effect on stress than on temperature. This is specially visible for the case of fast loading rates (Fig. 3b). The error on stress slightly increases with increasing $\alpha$, while the error on temperature decreases with $\alpha$ and is thus minimal for $\alpha=1$. In all cases, the dependence is very close to linear. From these results, it seems that the choice of a fully implicit approach for the temperature-dependence of visco-plastic parameters $(\alpha=1)$ could be recommended for obtaining the best precision on temperature without degrading too much the quality of stress values.

\section{Conclusion}

In this paper, we have shown how to construct a consistent incremental approximation of dissipation pseudo-potentials for the energy-based variational formulation of coupled thermo-viscoplasticity proposed in Yang et al. (2006) and Stainier and Ortiz (2010). We have seen that the most intuitive approximation did not lead to fully consistent results in the case of temperaturedependent yield and/or viscous stresses, and that an alternative approximation was thus needed. We have proposed such an approximation, ensuring fully consistent results, and have shown that it exhibits linear asymptotic convergence (with respect to time increment) of errors on stress and temperature. Such a linear convergence behavior was expected, since the variational incremental approach is globally an implicit backward-Euler scheme. The proposed consistent incremental approximation is parameterized by scalar coefficient $\alpha$, for which an optimal value $(\alpha=1)$ could be identified. This value indeed minimizes the error on the adiabatic temperature variation, while not significantly impacting associated values of stress. Due to the highly non-linear nature of thermomechanical coupling effects considered here, no real explanation could be obtained as for why this is the case (in more classical contexts, a mid-point rule often proves to be the most precise). A lead would be that this is probably linked to the explicit-implicit nature of factor $T_{n+1} / T_{n}$ which enters the formulation, and which is actually key to ensure its variational nature.

The example considered here was limited to a simple uniaxial tensile test, under adiabatic conditions. But the proposed consistent incremental approximation remains valid and applicable to more complex boundary-value problems, including conduction and other heat exchange mechanisms such as convection, within the variational framework described in Stainier (2009). And although we have considered in this paper the case of thermovisco-plasticity, the incremental approximation is also valid for other dissipative models, such as thermo-visco-elasticity, for example by extending the isothermal variational approach proposed in Fancello et al. (2006).

Finally, note that an alternative incremental variational formulation has recently been derived by Canadija and Mosler (2011), starting from the continuous formulation proposed in Yang et al. (2006). In a direct parallel to the continuous formulation, their approach uses an internal temperature field $\Theta$ which is determined independently of the external temperature field $T$. Factors $T_{n+1} / T_{n}$ are then replaced by $T_{n+1} / \Theta_{n+1}$, and $\Theta_{n+1}$ is also used as temperature parameter in the dissipation pseudo-potential. Consistency is then trivially verified. On the other hand, this formulation requires to use the internal energy instead of the free energy, and establishing analytical expressions for internal energy is significantly more complex in general cases than for free energy. The consistent incremental approach described here thus keeps all of its interest in such cases.

\section{References}

Argyris, J.H., Doltsinis, J.S., 1981. On the natural formulation and analysis of large deformation coupled thermo-mechanical problems. Computer Methods in Applied Mechanics and Engineering 25, 195-253.

Armero, F., Simo, J.C., 1992. A new unconditionally stable fractional step method for non-linear coupled thermomechanical problems. International Journal for Numerical Methods in Engineering 350 (4), 737-766.

Armero, F., Simo, J.C., 1993. A priori stability estimates and unconditionally stable product formula algorithms for nonlinear coupled thermoplasticity. International Journal of Plasticity 90 (6), 749-782.

Canadija, M., Mosler, J., 2011. On the thermomechanical coupling in finite strain plasticity theory with non-linear kinematic hardening by means of incremental energy minimization. International Journal of Solids and Structures 480 (7-8), 1120-1129.

Fancello, E., Ponthot, J.P., Stainier, L., 2006. A variational formulation of constitutive models and updates in non-linear finite viscoelasticity. International Journal for Numerical Methods in Engineering 65, 1831-1864.

Farhat, C., Park, K.C., Dubois-Pélerin, Y., 1991. An unconditionally stable staggered algorithm for transient finite element analysis of coupled thermoelastic problems. Computer Methods in Applied Mechanics and Engineering 85, 349-365.

Halphen, B., Nguyen, Q.S., 1975. Sur les matériaux standard généralisés. Journal de Mécanique 14, 39-63.

Oden, J.T., 1972. Finite Elements of Nonlinear Continua. McGraw-Hill, New-York.

Simo, J.C., Miehe, C., 1992. Associative coupled thermoplasticity at finite strains: formulation, numerical analysis and implementation. Computer Methods in Applied Mechanics and Engineering 98, 41-104.

Stainier, L., 2009. A variational finite element approach for thermo-mechanical coupling in computational structural mechanics. In: Schrefler, B., Oñate, E. Papadrakakis, M. (Eds.), COUPLED PROBLEMS 2009, International Conference on Computational Methods for Coupled Problems in Science and Engineering. Ischia, Italy.

Stainier, L., Ortiz, M., 2010. Study and validation of a variational theory of thermomechanical coupling in finite viscoplasticity. International Journal of Solids and Structures 470 (5), 705-715.

Yang, Q., Stainier, L., Ortiz, M.,2006. A variational formulation of the coupled thermomechanical boundary-value problem for general dissipative solids. Journal of the Mechanics and Physics of Solids 540 (2), 401-424. 